\title{
The Hypoglycaemic and Hypolipidemic Effects of Aqueous Extract of Alkanna strigosa in Alloxan Induced Diabetic Rats
}

\author{
Kamal Mansi ${ }^{1,}$,, Mariam Abu-al-basal ${ }^{1}$, Talal Aburjai ${ }^{2}$ \\ ${ }^{1}$ Department of Medical Laboratory Sciences, Faculty of Sciences, AL al-Bayt University, Al-Mafraq, Jordan \\ ${ }^{2}$ Faculty of Pharmacy, University of Jordan, Amman, Jordan
}

Email address:

mansikamal@hotmail.com (K. Mansi)

${ }^{*}$ Corresponding author

\section{To cite this article:}

Kamal Mansi, Mariam Abu-al-basal, Talal Aburjai. The Hypoglycaemic and Hypolipidemic Effects of Aqueous Extract of Alkanna strigosa in Alloxan Induced Diabetic Rats. Journal of Diseases and Medicinal Plants. Vol. 5, No. 4, 2019, pp. 60-68.

doi: $10.11648 /$ j.jdmp.20190504.11

Received: June 23, 2019; Accepted: July 18, 2019; Published: September 21, 2019

\begin{abstract}
Many plants have been used for the treatment of diabetes mellitus in Jordanian system of medicine and in other ancient systems of the world. The present study is aimed to evaluate the potential mechanism of antidiabetic action of aqueous extract of Alkanna strigosa aerial part and its hypolipidemic effects in normal and alloxan-induced diabetic rats. This study was conducted on fifty experimental animals. Adult albino rats (Sprague- Dawely strain) weighing about $220 \mathrm{~g}$ each were used throughout the study. Fifty rats were randomly assigned to five experimental groups of 10 rats each: Group 1 - received normal saline $(0.5 \mathrm{ml} / \mathrm{kg})$, and serves as control. Group II - gavaged daily for thirty days with $1 \mathrm{ml}$ of the extract at doses of $400 \mathrm{mg} / \mathrm{kg}$ body wt and served as control. Group III - Untreated diabetic rats that received two doses of alloxan $150 \mathrm{mg} / \mathrm{kg}$. Group IV Treated diabetic rats for thirty consecutive days with $1 \mathrm{ml}$ of the extract at a dose of $400 \mathrm{mg} / \mathrm{kg}$ body wt. Group V: Treated diabetic rats for thirty consecutive days with $14.2 \mathrm{mg} / \mathrm{kg}$ of metformin. Several hematological and biochemical parameters were assessed. It was found that the administration of aqueous extract of Alkanna strigosa produced significant reduction in blood glucose level in diabetic rats after thirty days of treatment. However, there was a significant $(p<0.05)$. Increase of insulin secretion. Also, the RBC and WBC count, PCV and neutrophil percentage decreased significantly $(\mathrm{p}<0.05)$. This study indicated that the aqueous extract of Alkanna strigosa increased the RBC and WBC counts, PCV, ESR, and neutrophil percentage in diabetic rats. However, the WBC count of the extract - treated diabetic group was still lower than those of control values. Administration of the extract resulted in a significant reduction in the mean values of serum cholesterol, triglyceride, LDL-C, ESR, urea, uric acid, creatinine accompanied by an increase in the mean values of total protein, albumin, insulin, HDL-C, neutrophile count and PCV in diabetic rats. No significant changes in these parameters were found in the control group. Effects produced by this extract were closely similar to a standard antidiabetic drug, metformin. $(\mathrm{p}<0.05)$ hypoglycemic effects in alloxan-induced diabetic rats, protection against body weight loss of diabetic animals and might alleviate diabetes-induced disturbances of some biochemical and hematological parameters. These results suggest that the oral administration of aqueous extract of the aerial part of Alkanna strigosa possesses antidiabetic and hypolipidemic effects in alloxan-induced diabetic rats.
\end{abstract}

Keywords: Alkanna strigosa, Antidiabetic, Hypolipidemic, Alloxan

\section{Introduction}

Many plants have been used for the treatment of diabetes mellitus in Jordanian system of medicine and in other ancient systems of the world. Diabetes is a chronic disorder in the metabolism of proteins, fats, and carbohydrates [1, 2]. It is described as an increase in blood glucose after any type of meal. Diabetes results from either insulin deficiency or malfunction [2]. According to statistics, $2.8 \%$ of the world's population suffer from this disease and it is expected to 
increase to more than $5.4 \%$ by 2025 [3] Diabetes is a disease that affects many people in the $21^{\text {st }}$ century and is known as the fifth leading cause to death [4]. There are several types of glucose-lowering drugs that exert anti-diabetic effects through different mechanisms. These mechanisms include stimulation of insulin secretion by sulfonylurea and meglitinides drugs, increasing of peripheral absorption of glucose by biguanides and thiazolidinediones [5], delay in the absorption of carbohydrates from the intestine by alphaglycosidase, and reduction of hepatic gluconeogenesis by biguanides [6].

Complex hormonal regulation of carbohydrate metabolism causes that presence of many endocrine disorders may disturb glucose homeostasis. Impaired fasting glucose, impaired glucose tolerance and frank diabetes are observed in patients with both common and rare endocrine disorders, particularly in patients with polycystic ovary syndrome [7], hyperthyroidism [8], Cushing's syndrome [9], pheochromocytoma, primary aldosteronism [10], acromegaly, growth hormone deficiency [11]. Because most of these disorders may be effectively treated and the treatment often results in a restoration of normal insulin secretion and receptor action as well as glucose absorption, production and metabolism [12], it is important to differentiate these disorders from other more common types of diabetes. This article reviews the etiology, clinical manifestation, diagnosis and management of endocrine disorders leading to diabetes and prediabetic states with special emphasis on the pathogenesis and clinical consequences of these disorders [13]. In the past three decades, despite the significant progress made in the treatment of diabetes, the results of treatment in patients is still far from perfect. These treatments have some disadvantages, including drug resistance (reduction of efficiency), side effects, and even toxicity. For example, sulfonylureas lose their effectiveness after 6 years of treatment in $44 \%$ of patients. It is also said that the glucoselowering drugs are not able to control hyperlipidemia [14]. In addition, the side effects of medicines and their interactions with each other in vitro must be considered by medical staff. Today, many treatments that involve the use of medicinal plants are recommended [15]. Most plants contain carotenoids, flavonoids, terpenoids, alkaloids, glycosides and can often have anti-diabetic effects [16]. The antihyperglycemic effects that results from treatment with plants are often due to their ability to improve the performance of pancreatic tissue, which is done by increasing insulin secretions or reducing the intestinal absorption of glucose.

The study [17] investigated the role of Acacia Arabica extract as a hypoglycemic, antihyperlipidemic, and antioxidant agent in streptozotocin-induced diabetic rats. The results found in this study indicate that Acacia Arabica extract has hypoglycemic, hypolipidemic, and antioxidant properties, therefore, it can be investigated for its efficacy in the treatment of diabetes in humans. Another study showed that the ethanol extract of Achyranthes aspera leaves (1000 $\mathrm{mg} / \mathrm{kg}$ ) used in STZ-induced diabetic rats significantly reduced their blood glucose level. This is probably due to the inhibition of glucose absorption from the intestine or because of an increase in glucose transport from the blood [18]. The hypoglycemic effects of water and butanolic extracts prepared from the bark of Acosmium panamense (Fabaceae) were studied in diabetic rats (streptozotocin (STZ)-induced). Oral application of water extracts at doses of 20 and 200 $\mathrm{mg} / \mathrm{kg}$ and of butanol extracts at doses of 20 and $100 \mathrm{mg} / \mathrm{kg}$ significantly lowered the plasma glucose levels in diabetic rats within $3 \mathrm{~h}$. Glibenclamide was used as reference and showed similar hypoglycemic effect like the extracts. Three structurally new compounds were isolated from the plant and shown to be the main constituents in both extracts. [19]. Aegle marmelos Corr. (Rutaceae) is widely used in Indian Ayurvedic medicine for the treatment of diabetes mellitus. The hypoglycaemic effect of the water extract of the fruits of Aegle marmelos was examined in streptozotocin-induced diabetic Wistar rats. Oral administration of the water extract (125 and 250mgkg (-1)) twice a day for 4 weeks resulted in significant reductions in blood glucose, plasma thiobarbituric acid reactive substances, hydroperoxides, ceruloplasmin and alpha-tocopherol and a significant elevation in plasma reduced glutathione and Vitamin $\mathrm{C}$ in diabetic rats. The effect of the extract at a dose of $250 \mathrm{mg} / \mathrm{kg}$ was more effective than glibenclamide in restoring the values of these parameters. The results of this study clearly show the hypoglycaemic activity of the fruit extract $[20,21]$, founded that the Oral administrations of the garlic extract significantly decreased serum glucose, total cholesterol, triglycerides, urea, uric acid, creatinine, AST and ALT levels, while increased serum insulin in diabetic rats but not in normal rats $(p<0.05)$. A comparison was made between the action of garlic extract and glibenclamide $(600 \mathrm{microg} / \mathrm{kg})$, the known antidiabetic drug. The antidiabetic effect of the extract was more effective than that observed with glibenclamide. Oral administration of A. squamosa aqueous extract to diabetic rats for 30 days significantly reduced the levels of blood glucose, lipids and lipid peroxidation, but increased the activities of plasma insulin and antioxidant enzymes, like catalase, superoxide dismutase, reduced glutathione and glutathione peroxidasehe A. squamosa aqueous extract supplementation is useful in controlling the blood glucose level, improves the plasma insulin, lipid metabolism and is beneficial in preventing diabetic complications from lipid peroxidation and antioxidant systems in experimental diabetic rats; therefore, it could be useful for prevention or early treatment of diabetes mellitus [22]. Artemisia herba Alba is widely used in Iraqi folk medicine for the treatment of diabetes mellitus. However, very few scientific and medical studies were carried out to assess the efficacy and toxicity of A. herba Alba. In this study feeding diabetic rats and rabbits with 0.39 $\mathrm{g} / \mathrm{kg}$ body weight of the aqueous extract of the aerial parts of the plant for 2-4 weeks shows a significant reduction in blood glucose level, prevents elevation of glycosylated haemoglobin level and possesses a hypoliposis effect, in addition to the protection against body weight loss of diabetic animals [23]. Alkanna tinctoria, the dyer's alkanet [24] or 
alkanet is well known in folk medicine and used for its antiinflammatory, anti-bacterial and wound healing properties [25]. A relevant species, A. strigosa, locally known as Hawa Jawi, is widely used in Jordan and surrounding area for the treatment of different ailments. A few studies have been conducted on this species, showing unremarkable properties. The plant was shown to be deprived of inhibitory activity on alpha-amylase of female rats [26] and the aqueous and methanol extracts of the plant revealed no significant antioxidant activity [27]. However, Oran reported an antitumor activity of the plant in Potato Disc Bioassay [28]. This study was dedicated to monitoring changes in the blood glucose and some hematological parameters in alloxaninduced diabetic rats under the hypoglycemic effects of aqueous extract of Alkanna strigosa in alloxan induced diabetic rats.

\section{Material and Methods}

\subsection{Plant Collection and Identification}

A. strigosa was collected locally in Jordan in March, 2018. The plant was taxonomically identified by Prof. Dawoud AlEisawi, a plant taxonomist, the Department of Biological Sciences, University of Jordan. Voucher specimen ( $A$. stigosa, 018) has been deposited at the Department of Pharmaceutical Sciences, Faculty of pharmacy, University of Jordan. The air-dried plant was ground to a fine texture and stored in a well closed container for further use.

\subsection{Preparation of the Extract}

Plant material was prepared according to the traditional method: The aerial part of Alkanna strigosa were powdered and dissolved in distilled water for $16 \mathrm{~h}$ with occasionally shaking each $2 \mathrm{~h}$. The extract was filtered. The filtrate was adjusted to concentration of $10 \mathrm{mg} \mathrm{mL}^{-1}$ of Alkanna strigosa.

\subsection{Animal Models}

This study was conducted in the experimental animal laboratory of the Faculty of Sciences, University of Jordan. All animals were housed, fed and treated in accordance with the in house guidelines for animal protection to minimize pain and discomfort. Adult male albino rats (Sprague-Dawely strain) weighing about $260 \mathrm{~g}$ each were used throughout the study. The animals were left for a week to adapt to the room conditions (temperature, humidity, light and dark period, aeration, and caging). Food and water were provided ad libitum. Animals were described as fasted were deprived of food for at least $12 \mathrm{~h}$ but were allowed free access to drinking water.

\subsection{Alloxan Induce Hyperglycemia}

Rats were made diabetic by injecting alloxan monohydrate "B. O. H chemical LTD England" intraperitoneally at a dose of $150 \mathrm{ml} / \mathrm{kg}$ (dissolved in fresh normal saline) to $18 \mathrm{~h}$ fasted rat. Every week after injection, blood was collected from the hearts of all surviving rats and blood glucose levels were determined. Rats with blood sugar levels of 200 to 450 $\mathrm{mg} / 100 \mathrm{ml}$ were considered as diabetic and were used in the study.

\subsection{Experimental Design}

The fifty rats were randomly assigned to 5 experimental groups of 10 rats each:

Group 1 -Received normal saline $(0.5 \mathrm{ml} / \mathrm{kg})$, and served as control.

Group II - Gavaged daily for thirty days with $1 \mathrm{ml}$ of the extract at doses of $400 \mathrm{mg} / \mathrm{kg}$ body wt and served as control.

Group III - Untreated diabetic rats that received two doses of alloxan $150 \mathrm{mg} / \mathrm{kg}$.

Group IV- Treated diabetic rats for thirty consecutive days with $1 \mathrm{ml}$ of the extract at a dose of $425 \mathrm{mg} / \mathrm{kg}$ body wt.

Group V- Treated diabetic rats for thirty consecutive days with $14.2 \mathrm{mg} / \mathrm{kg}$ of metformin. Metformin was purchased from Bristol - Myers Squibb Company, UK. The seed extract and metformin were daily given, using an intragastric tube for 6 wee ks. All rats were maintained in these treatment regimens for six weeks with free access to food and water. At the end of the experimental period, blood samples were taken from these experimental rats by cardiac puncture protocol. Rats were sacrificed by cervical dislocation under light ether anesthesia. These experiments complied with the guidelines of our animal ethics committee, which was established in accordance with the internationally accepted principles for laboratory animal use and care.

\subsection{Blood Sample Collection}

By the end of each experiment, the rats were reweighed, starved for 24 hours and sacrificed under chloroform anesthesia. $5 \mathrm{ml}$ of blood was collected from each animal by cardiac puncture using sterile needle and syringe. Part of the blood sample was put into test tubes and allowed to clot for 30 minutes before centrifuging using a bench top centrifuge (Cenformix). The remaining blood sample was put in an EDTA bottle for hematological determinations.

\subsection{Biochemical Analyses}

Serum glucose and lipid profile was determined after 12-14 h of fasting, including: Total Cholesterol (TC); triglycerides (TG); High-Density Lipoprotein Cholesterol (HDL-C) LowDensity Lipoprotein Cholesterol (LDL-C) by using commercial analytical kits from Sigma (St. Louis, Mo, USA).

\subsection{Hormonal Analyses}

Serum insulin and glucagon were measured through radioimmunoassay methods (CEA-JRE-SORIN Firm, France).

\subsection{Hematological Analyses}

The cell blood count (CBC) is usually performed on an automated hematology analyzer using whole well-mixed blood to which EDTA is added to prevent clotting. ESR was determined using Westengren method. Differential leukocyte 
count was conducted on Geimsa stained blood smears.

\subsection{Statistical Analyses}

The results were expressed as mean \pm standard deviation. Differences between control and experimental groups were estimated using students t-test analysis. Within group comparisons were performed by analysis of variance using ANOVA test. Differences were considered significant if Pvalue was less than 0.05 .

\section{Results}

Significant $(\mathrm{p}<0.05)$ weight loss was observed in untreated diabetic rats (group 3) than untreated normal rats (group 1) (Table 1). Treatment with aqueous extract of Alkanna strigosa (group 1) and $14.2 \mathrm{mg} / \mathrm{kg}$ of metformin respectively improved the weight gain compared to untreated to untreated diabetic rats (group 2). The blood glucose was increased significantly in untreated alloxan-induced diabetic rats (Table 2) as compared to untreated normal rats $(\mathrm{P} \leq 0.05)$. Administration of aqueous extract of Alkanna strigosa and $14.2 \mathrm{mg} / \mathrm{kg}$ of metformin respectively leads to significant $(\mathrm{P} \leq 0.05)$ decrease of the blood glucose levels in diabetics treated groups $(\mathrm{P} \leq 0.05)$. The administration of alloxan-induced diabetes significantly increased the glucose levels of the rats. The extract produced a significant decrease $(p<0.05)$ in blood glucose level in diabetic rats after 30 days of treatment by significantly increasing $(\mathrm{p}<$
$0.05)$ the secretion of insulin. None of the animals treated with extract showed any visible serious symptoms of toxicity; however, there were mild signs of respiratory distress, diarrhea, and convulsions. This indicates that aqueous extract of Alkanna strigosa may not cause any toxic effect on the body. The hypoglycemic effects of the aqueous extract on the diabetic rats were observed within $2 \mathrm{~h}$, continued for about $8 \mathrm{~h}$, and lasted to the end of the experiment ( 30 days). The average value of insulin was significantly lower $(\mathrm{p}<0.05)$ in the untreated diabetic rats compared to the respective control rats, as shown in (Table 3). On other hand, there were significant increases $(\mathrm{p}<$ 0.05 ) in the mean value of insulin in diabetic group treated with aqueous extract of Alkanna strigosa compared to the untreated diabetic rats during the entire period of the study. The insulin mean value in diabetic rats treated with metformin (Group V) was found to be closely similar to that in the untreated diabetic rats given only normal saline $(0.5 \mathrm{ml} / \mathrm{kg})$. Of note, the insulin mean value significantly reduced in the diabetic rats treated with metformin (Group V) relative to the control rats. Furthermore, the diabetic rats experienced significant increased $(p<0.05)$ in the mean value of glucagon when compared with the control rats; whereas the mean value of glucagon in the diabetic rats treated with aqueous extract of Alkanna strigosa (Group IV) or metformin was significantly $(\mathrm{p}<0.05)$ lower than that of the untreated diabetic rats. The insulin mean value was not significantly altered in the normal rats treated with the aqueous extract (Group II) relative to the control rats.

Table 1. Effect of aqueous extract of Alkanna strigosa on body weight in normal and alloxan induced diabetic rats after 30 days of treatment.

\begin{tabular}{lll}
\hline Groups & Initial weight $\mathbf{( g )}$ & Final weight \\
\hline I & $208 \pm 10.74$ & $241 \pm 9.48$ \\
II & $211 \pm 9.56$ & $250 \pm 18.66$ \\
III & $219 \pm 13.42$ & $196 \pm 7.54$ \\
IV & $204 \pm 6.83$ & $224 \pm 9.35$ \\
V & $218 \pm 16.62$ & $228 \pm 9.35$ \\
\hline
\end{tabular}

Values $(\mathrm{mg} / \mathrm{dL})$ are the mean values \pm standard deviation of 10 rats; *: Statistically significant when compared to control group (I) at $\mathrm{p}<0.05$; **: Statistically significant when compared to untreated diabetic group (III) at $\mathrm{p}<0.05$.

Table 2. Effect of aqueous extract of Alkanna strigosa on glucose, insulin ( $\mathrm{mg} / \mathrm{mL})$ and Glucagon (pg/mol) in normal and alloxan induced diabetic rats after 30 days of treatment.

\begin{tabular}{llll}
\hline Groups & Sugar $\mathbf{~ m g} / \mathbf{d l}$ & Insulin level $\boldsymbol{\mu} \mathbf{U} / \mathbf{m l}$ & Glucagon (pg/mol) \\
\hline I & $91.4 \pm 5.9$ & $5.85 \pm 1.64$ & $34.4 \pm 6.3$ \\
II & $89.8 \pm 4.8$ & $6.1 \pm 1.03$ & $36.8 \pm 4.4$ \\
III & $227.58^{* *} \pm 12.43$ & $3.83^{* *} \pm 1.6$ & $55.8 \pm 6.2^{* *}$ \\
IV & $198.78^{* *} \pm 11.19$ & $4.6 \pm 0.21^{* *}$ & $44.9 \pm 6.4^{* *}$ \\
V & $201.66^{* *} \pm 8.63$ & $4.1 \pm 0.8$ & $45.9 \pm 5.3^{* *}$ \\
\hline
\end{tabular}

Values $(\mathrm{mg} / \mathrm{dL})$ are the mean values \pm standard deviation of 10 rats; *: Statistically significant when compared to control group (I) at $\mathrm{p}<0.05 ; * *$ : Statistically significant when compared to untreated diabetic group (III) at $\mathrm{p}<0.05$.

Table 3. Effect of aqueous extract of Alkanna strigosa on glucose, Total protein, Albumin, Urea, Uric acid and Creatinine in normal and alloxan induced diabetic rats after 30 days of treatment.

\begin{tabular}{llllll}
\hline Group & Total protein $(\mathbf{g} / \mathbf{d L})$ & Albumin $(\mathbf{g} / \mathbf{d L})$ & Urea $(\mathbf{m g} / \mathbf{d L})$ & Uric acid $(\mathbf{m g} / \mathbf{d L})$ & Creatinine $(\mathbf{m g} / \mathbf{d L})$ \\
\hline I & $8.4 \pm 1.8$ & $3.6 \pm 0.8$ & $29.4 \pm 4.3$ & $1.7 \pm 0.6$ & $1.3 \pm 0.3$ \\
II & $7.9 \pm 1.4$ & $4.8 \pm 1.1^{*}$ & $29.2 \pm 6.6$ & $1.9 \pm 0.9$ & $1.5 \pm 0.5$ \\
III & $5.7 \pm 0.8^{*}$ & $1.7 \pm 0.7^{*}$ & $38.4 \pm 6.4$ & $2.7 \pm 1.0^{*}$ & $2.8 \pm 0.9^{*}$ \\
IV & $6.6 \pm 0.9^{* *}$ & $2.5 \pm 0.6^{* *}$ & $33.6 \pm 4.7$ & $1.8 \pm 0.4^{* *}$ & $1.6 \pm 0.5^{* *}$ \\
V & $7.1 \pm 1.7^{* *}$ & $3.2 \pm 0.4$ & $28.8 \pm 5.8$ & $1.7 \pm 0.4^{* *}$ & $1.5 \pm 0.6^{* *}$ \\
\hline
\end{tabular}

Values $(\mathrm{mg} / \mathrm{dL})$ are the mean values \pm standard deviation of 10 rats; *: Statistically significant when compared to control group (I) at $\mathrm{p}<0.05 ; * *$ : Statistically significant when compared to untreated diabetic group (III) at $\mathrm{p}<0.05$. 
As can be seen in (Table 4) the mean values of urea, uric acid and creatinine were significantly higher in the untreated diabetes rats as compared to the control rats $(\mathrm{p}<0.05)$. Treatment of the diabetic rats with aqueous extract of Alkanna strigosa and metformin for 30 days (Groups IV and V) caused a significant decrease in urea, uric acid and creatinine as compared to the untreated diabetic group ( $\mathrm{p}<0.05)$. In addition, the average values of total protein and albumin levels in the untreated diabetic rats were significantly lower $(p<0.05)$ than that of the control rats. On other hand, in the diabetic groups treated with aqueous extract of Alkanna strigosa or metformin, the average values of total protein and albumin levels significantly $(\mathrm{p}<0.05)$ increased, as compared with those of the untreated diabetic group.

Table 4. Effect of aqueous extract of Alkanna strigosa on serum lipid profiles in normal and alloxan induced diabetic rats after 30 days of treatment.

\begin{tabular}{|c|c|c|c|c|}
\hline Group & Total Cholesterol & Triglyceride & HDL-C & LDL-C \\
\hline $\mathrm{I}$ & $126 \pm 9.43$ & $73 \pm 12.4$ & $33 \pm 6.1$ & $31 \pm 5.8$ \\
\hline II & $118 \pm 12.62$ & $86 \pm 7.9$ & $32 \pm 4.3$ & $28 \pm 4.7$ \\
\hline III & $189 \pm 24.45^{*}$ & $132 \pm 21.7 *$ & $24 \pm 3.8^{*}$ & $54 \pm 12.6^{*}$ \\
\hline V & $147 \pm 18.7 * *$ & $97 \pm 19.4 * *$ & $28 \pm 4.2 * *$ & $39 \pm 10.6^{* *}$ \\
\hline
\end{tabular}

Values $(\mathrm{mg} / \mathrm{dL})$ are the mean values \pm standard deviation of 10 rats; $*$ : Statistically Significant when compared to control group (I) at $\mathrm{p}<0.05 ; * *$ Statistically significant when compared to untreated diabetic group (III) at $\mathrm{p}<0.05$.

Serum total cholesterol, triglycerides, LDL cholesterol and (TC/HDL) were significantly elevated in untreated diabetic rats as compared to untreated normal rats $(\mathrm{p}<0.05)$. All lipids parameters tested were improved after the treatment with aqueous extract of Alkanna strigosa and metformin. No effect was observed in treated normal rats (Table 5).
The administration of water extracts of aqueous extract of Alkanna strigosa significantly increased $((\mathrm{p}<0.05) \mathrm{RBC}$, $\mathrm{PCV}, \mathrm{ESR}$, and neutrophil percentage in diabetic rats. However, the WBC count of the A. graveolens seed extract treated diabetic group was still lower than those of control values (Table 6).

Table 5. Effect of aqueous extract of Alkanna strigosa on levels of some hematological parameters in normal and alloxan induced diabetic rats after 30 days of treatment.

\begin{tabular}{|c|c|c|c|c|c|}
\hline \multirow{2}{*}{ Parameter } & Group & Group & Group & Group & Group \\
\hline & I & II & III & IV & $\mathbf{V}$ \\
\hline $\mathrm{Hb}(\mathrm{g} / \mathrm{dL})$ & $12.3 \pm 1.8$ & $12.5 \pm 1.6$ & $10.9 \pm 1.6$ & $11.9 \pm 0.4$ & $12.1 \pm 1.9$ \\
\hline RBCs $x 106 / \mu \mathrm{L}$ & $5.4 \pm 0.8$ & $5.6 \pm 0.8$ & $4.4 \pm 0.7$ & $5.1 \pm 0.8$ & $5.3 \pm 1.1$ \\
\hline WBCs $\times 103 / \mu$ & $7.7 \pm 1.8$ & $6.8 \pm 1.2$ & $4.2 \pm 1.0$ & $3.8 \pm 0.4$ & $3.7 \pm 0.6$ \\
\hline Neutrophils \% & $34.6 \pm 3.3$ & $35.8 \pm 4.2$ & $26.3 \pm 5.7 *$ & $36.8 \pm 4.1^{* *}$ & $36.7 \pm 2.9 * *$ \\
\hline Basophiles \% & $2.4 \pm 0.4$ & $2.5 \pm 0.6$ & $3.1 \pm 0.5$ & $3.4 \pm 0.2$ & $3.3 \pm 0.7$ \\
\hline Eosinophils \% & $3.8 \pm 0.4$ & $4.2 \pm 1.1$ & $4.4 \pm 0.6$ & $4.3 \pm 0.2$ & $4.8 \pm 1.22$ \\
\hline $\mathrm{PCV}$ & $38.5 \pm 2.9$ & $37.5 \pm 2.6$ & $30.7 \pm 2.3 *$ & $36.7 \pm 2.9 * *$ & $35.8 \pm 3.9 * *$ \\
\hline ESR & $13.8 \pm 2.4$ & $11.7 \pm 2.1$ & $20.6 \pm 2.4^{*}$ & $15.8 \pm 2.2 * *$ & $14.6 \pm 3.7 * *$ \\
\hline
\end{tabular}

Values $(\mathrm{mg} / \mathrm{dL})$ are the mean values \pm standard deviation of 10 rats; $*$ : Statistically significant when compared to control group (I) at $\mathrm{p}<0.05 ; * *$ : Statistically significant when compared to untreated diabetic group (III) at $\mathrm{p}<0.05$.

\section{Discussion}

Our study clearly demonstrated ant hyperglycemic action of the aqueous extract of Alkanna strigosa. Thus, an attempt has been made in the current study to investigate the potential mechanism of antidiabetic action of this extract in alloxan induced diabetic rats. Alloxan induces diabetes by damaging the insulin secreting cells of the pancreas leading to hyperglycemia [29]. An observation in this study correlates with the previous research finding, in that the blood glucose levels significantly increased in alloxan untreated diabetic rats [30]. Alloxan induces damage and death of pancreatic islet-cells in several experimental animal models, thus causing diabetes mellitus and decreasing the secretion of insulin [31]. The cytotoxic action of this diabetogenic agent is mediated by reactive oxygen species, Alloxan and the product of its reduction, dial uric acid; establish a redox cycle with the formation of super oxide radicals [31]. These radicals undergo dismutation to hydrogen peroxide [31]. Thereafter highly reactive hydroxyl radicals are formed by the Fenton reaction [32]. The action of reactive oxygen species with a simultaneous massive increase in cytosolic calcium concentration causes rapid destruction of $\beta$-cells [31]. The blood glucose data obtained clearly indicate that aqueous extract from Alkanna strigosa produced significant hypoglycemic effects in alloxan-induced diabetic rats. It is possible that the plant may reverse the catabolic features of insulin deficiency, decrease the release of glucagon or increase that of insulin, stimulate directly glycolysis in peripheral tissues, increase glucose removal from blood or reduce glucose absorption from the gastrointestinal tract [33]. Hypoglycemic effects of Alkanna strigosa extract could, possibly, be due to increased peripheral glucose utilization. Inhibition of the proximal tubular reabsorption mechanism for glucose in the kidneys, if any, can also contribute towards blood lowering effect [34]. Body weight in all diabetic rats 
was increased. This is the normal effect of diabetes mellitus. After the treatment of the diabetic rats, their body weight increased again. Similar effects were also observed by other researchers [35-37]. The synthetic oral hypoglycemic agents can produce a series of side effects. As can be seen from the study, rats treated with Alkanna strigosa showed only mild visible undesirable clinical symptoms. We have noticed a significant reduction in food and water intake in alloxan diabetic rats. This could be the result of improved glycaemic control produced by aqueous extract of Alkanna strigosa.

The present study indicated that the aqueous extract of Alkanna strigosa or metformin treatment might ameliorate some disturbed hematological parameters of diabetic rats. It has been suggested that anemia occurrence in DM is due to the increased non-enzymatic glycosylation of $\mathrm{RBC}$ membrane proteins, which correlates with hyperglycemia [38]. Oxidation of these glycosylated membrane proteins and hyperglycemia in DM caused an increase in the production of lipid peroxides causing the hemolysis of RBC [39]. In this study, the RBC membrane lipid peroxide levels in diabetic rats were not measured. However, [40], demonstrated that serum lipid peroxide level increased in diabetic rabbits. Thus, increased RBC count of aqueous extract of Alkanna strigosa or metformin treatment rats could be due to the lowered lipid peroxide level in RBC membrane leading to decreased susceptibility of RBC to hemolysis. Since nonenzymatic glycosylations of membrane proteins correlate with hyperglycemia by measuring the RBC fragility, and serum folic acid, iron, cobalt, vitamin B12, and calcium levels are needed to demonstrate the exact mechanism of action of aqueous extract of Alkanna strigosa or metformin on increased RBC count of diabetic rats. Neutrophils ingest and kill bacteria and have been called the body's first line of defense against bacterial infections [41]. It has been postulated that the body's defense mechanism against infections was disturbed due to the disturbed neutrophil function in diabetes [42]. In this study, we demonstrated that aqueous extract of Alkanna strigosa or metformin treatment increased the lowered neutrophil percentage of WBC to the level of control. This result indicated aqueous extract of Alkanna strigosa or metformin treatment might also increase the defense mechanism of the body against infections. In diabetic rats, Alloxan-induced diabetes increased the heart rate while aqueous extract of Alkanna strigosa or metformin treatment decreased it to control level. The increased heart rate in diabetic rats was probably due to the increased sympathetic output produced by diabetes-induced anemia. In the present study, it was found that the heart rate decreased and also RBC count increased to control level in aqueous extract of Alkanna strigosa or metformin treated rats. Therefore, decreased heart rate could also be due to a normalized RBC count in these rats. We have noticed elevated serum lipids in alloxan-diabetic rats. Lipids play an important role in the pathogenesis of diabetes mellitus. The level of serum lipids is usually raised in diabetes and such an elevation represents a risk factor for coronary heart disease [43]. Lowering of serum lipids levels through dietary or drugs therapy seems to be associated with a decrease in the risk of vascular disease [44]. The abnormal high concentration of serum lipids in diabetes is mainly due to the increase in the mobilisation of fatty acids from the peripheral depots, since insulin inhibits the hormone sensitive lipase. On the other hand, glucagon, catecholamines and other hormones enhance lipolysis. The marked hyperlipidemia that characterizes the diabetic state may therefore be regarded as a consequence of the uninhibited actions of lipolytic hormones on the fat depots [45]. In our study, we have also observed an increase in the concentration of total cholesterol, triglycerides, LDL cholesterol and TC/HDL-C in alloxan untreated diabetic rats. Hyperlipidemia is a recognized consequence of diabetes mellitus [46, 47]. Administration aqueous extract of Alkanna strigosa or metformin treatment normalized serum lipids, secondary to the diabetic state. Diabetes-induced hyperlipidemia is attributable to excess mobilization of fat from the adipose due to the under utilization of glucose [48]. The ability of aqueous extract of Alkanna strigosa reduce the levels of plasma lipids in diabetic rats by the increasing the utilization of glucose, thereby depressing the mobilization of fat. In addition to marked hyperglycemia, our result revealed that the alloxaninduced diabetic rats developed notable hyperlipidaemia. Diabetes-induced hyperlipidaemia was observed in diabetic experimental animal models, and it is associated with an increase of mobilization of fat from fat cells and lipid metabolism due to the inability to utilize glucose properly $[49,50]$. This is very important since elevated concentrations of cholesterol, triglyceride, and LDL-C are important risk factors in the development of arterioscleroses in diabetes mellitus. Administration of aqueous extract of Alkanna strigosa or metformin normalized serum lipids, secondary to the diabetic state. Diabetes-induced hyperlipidemia is attributable to the excess mobilization of fat from the adipose due to the underutilization of glucose [51]. The ability of aqueous extract of Alkanna strigosa or metformin reduces the levels of plasma lipids in diabetic rats by increasing the utilization of glucose, thereby depressing the mobilization of fat. Our findings are consistent with a recent study by [52], which reported that leaves of Leucas cephalotes lowered both plasma and hepatic lipid profiles (total lipid, triglycerides, and cholesterol) and LDL-C while elevating the HDL-C levels [53]. They suggest that these improvements in lipid profiles are most likely due to its insulin-like actions of the leaves extract. It is possible to suggest that the mechanism (s) of antihyperlipidemic effect of the aqueous extract of Alkanna strigosa might be similar to some of those suggested for anti-diabetic plants exhibiting antihyperlipidemic activity, such as activation of lipoprotein lipase, insulin-mediated lipolytic activity or inhibition of lipogenic enzymes or hormone-sensitive lipase [54]. Similar results were observed in the effect of ethanol extract of Iris germanica L. rhizomes (Iridaceous), they indicated that ethanol extract of Iris germanica has remarkably lowered the lipid components, particularly, the cholesterol and triglycerides [55]. The results suggest that the lipid-lowering action of this natural 
product may be mediated through inhibition of hepatic cholesterol biosynthesis, increased faucal bile acids excretion, and enhanced plasma lecithin: cholesterol acyltransferase activity, and reduction of lipid absorption in the intestine. Our study also showed a significant decrease in serum total protein and albumin in untreated diabetic rats, whereas total protein and albumin significantly increased after the administration of this extract. The total protein and albumin levels in the blood can also be used as an indicator of liver function. Similar results were obtained when the metformin was administered orally in alloxan diabetic. These results suggest that this extract can improve some biochemical parameters that are related to liver functions. Hyperglycemia has also been recently implicated in the initiation and development of various types of diabetic complications. Nephropathy is one of these serious microvascular complications that have been observed in diabetic individuals [56]. In addition, blood urea and creatinine concentrations were increased among uncontrolled diabetic individuals and this increase could be a result of impaired renal function due to an increased blood glucose level. Our results revealed for the first time that the mean values of these end products in that serum increased in untreated diabetic rats, while they significantly decreased after the administration of extracts. Thus, this extract might improve renal function which, in turn, leads to a reduction in these end products. It was reported that diabetic individuals had lower serum albumin concentrations as well as higher serum uric acid and urea levels than nondiabetic individuals [56]. Thus, the reduction in urea and creatinine levels probably can be explained by a reduction in blood glucose level. In conclusion, the present study indicates that the aqueous extract of Alkanna strigosa or metformin to exhibit hypoglycemic and hypolipidemic activities in alloxan-induced diabetic rats.

\section{Conclusion}

In conclusion, this is the first study to reveal that oral administration of aqueous extract of Alkanna strigosa enhances insulin production and decreases glucagon production in the alloxan-induced diabetic rats. Thus, oral use of this extract might positively affect the functional capacities of various rat tissues, particularly blood, heart, kidney and liver against toxic action of alloxan compound (dose of $150 \mathrm{mg} / \mathrm{Kg} \mathrm{BW}$ ). These findings clearly support the traditional use of this medicinal plant in treatment of diabetes mellitus and shed more light on the efficacy of this plant. Thus, Alkanna strigosa to be a valuable plant and ideally suited to be used in treatment of DM.

\section{Acknowledgements}

The support from Al al-Bayet University and is gratefully acknowledged. We also would thank Dr: Talal Aburjai for his assistance in using his research lab in the Faculty of Pharmacy at the University of Jordan.

\section{References}

[1] Osadebe PO, Odoh EU, Uzor PF. The search for new hypoglycemic agents from plant. Afr J Pharm Pharmacol. 2014; 8 (11): 292-303. doi: 10.5897/AJPP2014.3933.

[2] Modak M, Dixit P, Londhe J, Ghaskadbi S, Devasagayam TPA. Indian herbs and herbal drugs used for the treatment of diabetes. J Clin Biochem Nutr. 2007; 40 (3): 163. doi: $10.3164 /$ jcbn. 40.163 .

[3] Mukesh R, Namita P. Medicinal Plants with Antidiabetic Potential-A Review. American-Eurasian J Agric Environ Sci. 2013; 13 (1): 81-94.

[4] Kazi S. Use of traditional plants in diabetes mellitus. Int J Pharm. 2014; 4 (4): 2839.

[5] Bathaie S, Mokarizade N, Shirali S. An overview of the mechanisms of plant ingredients in the treatment of diabetes mellitus. J Med Plant. 2012; 4 (44): 1-24.

[6] Hui H, Zhao X, Perfetti R. Structure and function studies of glucagon-like peptide-1 (GLP1): the designing of a novel pharmacological agent for the treatment of diabetes. Diabetes Metab Res Rev. 2005; 21: 313-31. doi: 10.1002/dmrr.553.

[7] De Leo V, Musacchio MC, Morgante G, La Marca A, Petraglia F. olycystic ovary syndrome and type 2 diabetes mellitus. Minerva Ginecol. 2004 Feb; 56 (1): 53-62.

[8] Mirella Hage, Mira S. Zantout, and Sami T. Azar. Thyroid Disorders and Diabetes Mellitus. J Thyroid Res. 2011; 2011; 439463. Published online 2011 Jul 12. doi: $10.4061 / 2011 / 439463$.

[9] Mattia Barbot, Filippo Ceccato, and Carla Scaroni. Diabetes Mellitus Secondary to Cushing's disease. Front Endocrinol (Lausanne). 2018; 9: 284.

[10] Young WF Jr. Adrenal causes of hypertension: pheochromocytoma and primary aldosteronism. Rev Endocr Metab Disord. 2007 Dec; 8 (4): 309-20.

[11] Francesco Ferraù Adriana Albani Alessandro Ciresi Carla Giordano and Salvatore Cannavò. Diabetes Secondary to Acromegaly: Physiopathology, Clinical Features and Effects of Treatment. Front Endocrinol (Lausanne). 2018; 9: 358.

[12] Gisela Wilcox, insulin and Insulin Resistance. Clin Biochem Rev. 2005 May; 26 (2): 19-39.

[13] Yanling Wu' Yanping Ding' Yoshimasa Tanaka' and Wen Zhang. Risk Factors Contributing to Type 2 Diabetes and Recent Advances in the Treatment and Prevention Int J Med Sci. 2014; 11 (11): 1185-1200.

[14] Dey L, Attele AS, Yuan CS. Alternative therapies for type 2 diabetes. Altern Med Rev. 2002; 7: 45-58.

[15] Kooti W, Moradi M, Akbari SA, Sharafi-Ahvazi N, AsadiSamani M, Ashtary-Larky D. Therapeutic and pharmacological potential of Foeniculum vulgare Mill: A review. J HerbMed Pharmacol. 2015; 4: 1-9.

[16] Afrisham R, Aberomand M, Ghaffari MA, Siahpoosh A, Jamalan M. Inhibitory Effect of Heracleum persicum and Ziziphus jujuba on Activity of Alpha-Amylase. Journal of Botany. 2015; 2015: 1-8. doi: 10.1155/2015/824683. 
[17] Hegazy GA, Alnoury AM, Gad HG. The role of Acacia Arabica extract as an antidiabetic, antihyperlipidemic, and antioxidant in streptozotocin-induced diabetic rats. Saudi Med J. 2013 Jul; 34 (7): 727-33.

[18] Kumar A, Gnananath K, Gande S, Goud E, Rajesh P, Nagarjuna S. Anti-diabetic Activity of Ethanolic Extract of Achyranthes aspera Leaves in Streptozotocin induced diabetic rats. Journal of Pharmacy Research. 2011; 4: 3124-5.

[19] Andrade-Cetto A, Wiedenfeld H. Hypoglycemic effect of Acosmium panamense bark on streptozotocin diabetic rats. J Ethnopharmacol. 2004 Feb; 90 (2-3): 217-20.

[20] Zhang X-F, Tan B. Anti-diabetic property of ethanolic extract of Andrographis paniculata in streptozotocin-diabetic rats. Acta Pharmacol Sin. 2000; 21 (12): 1157-64.

[21] Eidi A, Eidi M, Esmaeili E. Antidiabetic effect of garlic (Allium sativum L.) in normal and streptozotocin-induced diabetic rats. Phytomedicine. 2006; 13 (9): 624-9. DOI 10.1016/j.phymed.2005.09.010

[22] Kaleem M, Asif M, Ahmed Q, Bano B. Antidiabetic and antioxidant activity of Annona squamosa extract in streptozotocin-induced diabetic rats. Singapore Med J. 2006; 47 (8): 670-5.

[23] Raina R, Prawezi S, Verma PK, Pankaj NK. Medicinal plants and their role in wound healing. Vet Scand. 2008; 3: 1-7.

[24] Talib WH, Mahasneh AM. Antimicrobial, cytotoxicity and phytochemical screening of Jordanian plants used in traditional medicine. Molecules. 2010; 15: 1811-24. doi: 10.3390/molecules 15031811 .

[25] Morton JF. Search for carcinogenic principles. In: Swaain T, Kleiman R, editors. Recent advances in phytochemistry. Florida, USA: Plenum Press; 1980.

[26] Imad I. Hamdan, Fatma U. Afifi, Screening of Jordanian flora for $\alpha$-amylase inhibitory activity, Pharm. Biol. 46 (10-11) (2008) 746-750.

[27] Feras Q. Alali, Khaled Tawaha, Tamam El-Elimat, Maha Syouf, Mosa El-Fayad, Khaled Abulaila, Samara Joy Nielsen, William D. Wheaton, Joseph O. Falkinham III, Nicholas H. Oberlies, Antioxidant activity and total phenolic content of aqueous and methanolic extracts of Jordanian plants: an ICBG project, Nat. Prod. Res. 21 (12) (2007) 1121-1131.

[28] Sawsan A. Oran, Potato disc bioassay for some Jordanian medicinal plants, Pharm. Biol. 37 (4) (1999) 296-299.

[29] Szkudelski, T., 2001. The mechanism of alloxan and streptozotocin action in $\beta$ cells of the rat pancreas. Physiol. Res., 50: 537-546.

[30] Amanda Natália Lucchesi' Lucas Langoni Cassettariand César Tadeu Spadella. Alloxan-Induced Diabetes Causes Morphological and Ultrastructural Changes in Rat Liver that Resemble the Natural History of Chronic Fatty Liver Disease in Humans. J Diabetes Res. 2015; 2015: 494578.

[31] Ankur Rohilla and Shahjad Ali. Alloxan Induced Diabetes: Mechanisms and Effects. International Journal of Research in Pharmaceutical and Biomedical Sciences. Vol. 3 (2) Apr - Jun 2012 www.ijrpbsonline.com.

[32] Alugoju Phaniendra, Dinesh Babu Jestadi, and Latha
Periyasamy. Free Radicals: Properties, Sources, Targets, and Their Implication in Various Diseases. Indian J Clin Biochem. 2015 Jan; 30 (1): 11-26.

[33] Didem taştekin, mustafa atasever, gülşah adigüzel, mustafa keleş and ayhan taştekin. Hypoglycaemic effect of artemisia herba-alba in experimental hyperglycaemic rats. Bull Vet Inst Pulawy 50, 235-238, 2006.

[34] Algandaby MM, Alghamdi HA, Ashour OM, Abdel-Naim AB, Ghareib SA, Abdel-Sattar EA, Hajar AS. Mechanisms of the antihyperglycemic activity of Retama raetam in streptozotocin-induced diabetic rats. Food Chem Toxicol. $2010 \quad$ Aug-Sep; $48 \quad$ (8-9): 2448-53. doi: 10.1016/j.fct.2010.06.01.

[35] Ramya Rajasekar, Kalaiselvi Manokaran, Narmadha Rajasekaran, Gomathi Duraisamy, andDevaki Kanakasabapathi. Effect of Alpinia calcarata on glucose uptake in diabetic rats-an in vitro and in vivo model. $\mathrm{J}$ Diabetes Metab Disord. 2014; 13: 33.

[36] Nasser S. Abou Khalil Alaa S. Abou-Elhamd, Salwa I. A. Wasfy, Ibtisam M. H. El Mileegy, Mohamed Y. Hamed, and Hussein M. Ageely. Antidiabetic and Antioxidant Impacts of Desert Date (Balanites aegyptiaca) and Parsley (Petroselinum sativum) Aqueous Extracts: Lessons from Experimental Rats. J Diabetes Res. 2016; 2016: 8408326.

[37] Dan Tang, Liu Liu, Dildar Ajiakber, Jianping Ye, Jianjun Xu, Xuelei Xin and Haji Akber Aisa, Anti-diabetic Effect of Punica granatum Flower Polyphenols Extract in Type 2 Diabetic Rats: Activation of Akt/GSK-3 $\beta$ and Inhibition of IRE1 $\alpha$-XBP1 Pathways. Front. Endocrinol., 15 October 2018 | https://doi.org/10.3389/fendo.2018.00586.

[38] Ayman M. Mahmoud. Hematological alterations in diabetic rats - Role of adipocytokines and effect of citrus flavonoids. EXCLI J. 2013; 12: 647-657.

[39] Jana Viskupicova, Dusan Blaskovic, Sabina Galiniak, Mirosław Soszyński, Grzegorz Bartosz, Lubica Horakova, and Izabela Sadowska-Bartosz. Effect of high glucose concentrations on human erythrocytes in vitro. Redox Biol. 2015 Aug; 5: 381-387.

[40] Jaouhari JT, Lazrek HB, Jana M.. The hypoglycemic activity of Zygophyllum gaetulum extracts in alloxan-induced hyperglycemic rats. J Ethnopharmacol. 2000 Jan; 69 (1): 1720.

[41] Cavalher-Machado SC, de Lima WT, Damazo AS, de Frias Carvalho V, Martins MA, Silva PM, et al, 2004. Downregulation of mast cell activation and airway reactivity in diabetic rats: role of insulin. Eur Respir J; 24: 552-558.

[42] Moriguchi P, Sannomiya P, Lara PF, Oliveira-Filho RM, Greco KV, Sudo-Hayashi LS. 2005. Lymphatic system changes in diabetes mellitus: role of insulin and hyperglycemia. Diabetes Metab Res Rev; 21: 150-157.

[43] Benjamin M Leon and Thomas M Maddox. Diabetes and cardiovascular disease: Epidemiology, biological mechanisms, treatment recommendations and future research. World J Diabetes. 2015 Oct 10; 6 (13): 1246-1258.

[44] K Mahdy Ali, A Wonnerth, K Huber, and J Wojta. Cardiovascular disease risk reduction by raising HDL cholesterol - current therapies and future opportunities. Br J Pharmacol. 2012 Nov; 167 (6): 1177-1194. 
[45] S. Radhika, K. H. Smila and R. Muthezhilan. Antidiabetic and Hypolipidemic Activity of Punica granatum Linn on Alloxan Induced Rats. World Journal of Medical Sciences 6 (4): 178$182,2011$.

[46] Pushparaj, P., C. H. Tan and B. K. H. Tan, 2000. Effects of Averrhoa bilimbi leaf extract on blood glucose and lipids in streptozotocin-diabetic rats. J. Ethnopharmacol., 72: 69-76.

[47] Pepato, M. T., A. M. Baviera, R. C. Vendramini, M. P. Perez, I. C. Kettelhut and I. L. Brunetti, 2003. Cissus sicyoides (Princess wine) in the long term treatment of streptozotocindiabetic rats. Biotechnol. Applied Biochem., 37: 15-20.

[48] Enomfon J Akpan, Jude E Okokon, and Emem Offong Antidiabetic and hypolipidemic activities of ethanolic leaf extract and fractions of Melanthera scandens. Asian Pac J Trop Biomed. 2012 Jul; 2 (7): 523-527.

[49] Raz I, Eldor R, Cernea S, Shafrir E. Diabetes: insulin resistance and derangements in lipid metabolism. Cure through intervention in fat transport and storage. Diabetes Metab Res Rev. 2005 Jan-Feb; 21 (1): 3-14.

[50] Sachdewa A, Khemani LD. Effect of Hibiscus rosa sinensis Linn. Ethanol flower extract on blood glucose and lipid profile in streptozotocin induced diabetes in rats. J Ethnopharmacol. 2003 Nov; 89 (1): 61-6.

[51] Krishna, B., S. Nammi, M. K. Kota and R. V. Krishna Rao, 2004. Evaluation of hypoglycemic and antihyperglycaemic effects of Datura metel Linn. seeds in normal and alloxaninduced diabetic rats. J. Ethnopharmacol., 9: 95-98.
[52] Nagappa, A. N., P. A. Thakurdesai, N. V. Rao and J. Singh, 2003. Antidiabetic activity of Terminalia catappa Linn fruits. J. Ethnopharmacol., 88: 45-50.

[53] Ramkumar KM, Latha M, Venkateswaran S, Pari L, Ananthan R, Bai VN. Modulatory effect of Gymnema montanum leaf extract on brain antioxidant status and lipid peroxidation in diabetic rats. J Med Food. 2004 Fall; 7 (3): 366-71.

[54] Ruzaidi A, Amin I, Nawalyah AG, Hamid M and Faizul HA, 2005. The effect of malaysian cocoa extract on glucose levels and lipid profiles in diabetic rats. J. Ethnopharmacol. 98: 5560 .

[55] Choudhary MI, Naheed S, Jalil S, Alam JM, Atta-ur-Rahman., Effects of ethanolic extract of Iris germanica on lipid profile of rats fed on a high-fat diet. J Ethnopharmacol. 2005 Apr 8; 98 (1-2): 217-20.

[56] Aastha Chawla, Rajeev Chawla, and Shalini Jaggi Microvasular and macrovascular complications in diabetes mellitus: Distinct or continuum? Indian J Endocrinol Metab. 2016 Jul-Aug; 20 (4): 546-551.

[57] Ahmed I, Lakhani MS, Gillett M, John A, Raza H. Hypotriglyceridemic and hypocholesterolemic effects of antidiabetic Momordica charantia Karela. Fruit extract in streptozotocin-induced diabetic rats. Diabet. Res. Clin. Pract. 2001; 51: 155-161. 